\title{
D-Finder 2: Towards Efficient Correctness of Incremental Design
}

\author{
Saddek Bensalem ${ }^{1}$, Andreas Griesmayer ${ }^{1}$, Axel Legay ${ }^{3}$, Thanh-Hung Nguyen ${ }^{1}$, \\ Joseph Sifakis ${ }^{1}$, and Rongjie Yan ${ }^{1,2}$ \\ 1 Verimag Laboratory, Université Joseph Fourier Grenoble, CNRS \\ 2 State Key Laboratory of Computer Science, Institute of Software, CAS, Beijing \\ 3 INRIA/IRISA, Rennes
}

\begin{abstract}
D-Finder 2 is a new tool for deadlock detection in concurrent systems based on effective invariant computation to approximate the effects of interactions among modules. It is part of the BIP framework, which provides various tools centered on a component-based language for incremental design. The presented tool shares its theoretical roots with a previous implementation, but was completely rewritten to take advantage of a new version of BIP and various new results on the theory of invariant computation. The improvements are demonstrated by comparison with previous work and reports on new results on a practical case study.
\end{abstract}

\section{Context}

Language. D-Finder 2 is part of a framework of tools that share a common language, $B I P$, to describe component-based systems [1]. The language is based on atomic components and connectors to describe their interactions. Components can also be hierarchically organized to build new components. An atomic component is a transition system $B=(L, P, \mathcal{T})$, where $L=\left\{l_{1}, l_{2}, \ldots, l_{k}\right\}$ is a set of control locations, $P$ is a set of ports, and $\mathcal{T} \subseteq L \times P \times L$ is a set of transitions. A component additionally can contain data and use $\mathrm{C}$ code for actions and conditions on the transitions to manipulate this data. Figure 1 shows a graphical representation of two atomic components $B_{1}$ and $B_{2}$. We use cycles for locations and arrows for transitions. Every transition is labeled by a port to synchronize with ports of other components to create interactions. In the example, the ports trigger and tick of the two components are synchronized, which means the corresponding transitions have to be executed concurrently, and are only available if the guards in both components are fulfilled. The transition rel can be taken whenever a component is in the fire location. We can give only a very brief description of $B I P$ here, please refer to [1] for more details.

Verification. Previous work 3/4 introduced an efficient verification method for the models above. Key to this method is the approximation of the reachable states by compositional invariant computation based on (1) component invariants $\Phi_{i}$ that capture the constraints on local data of a component $B_{i}$, and (2) 


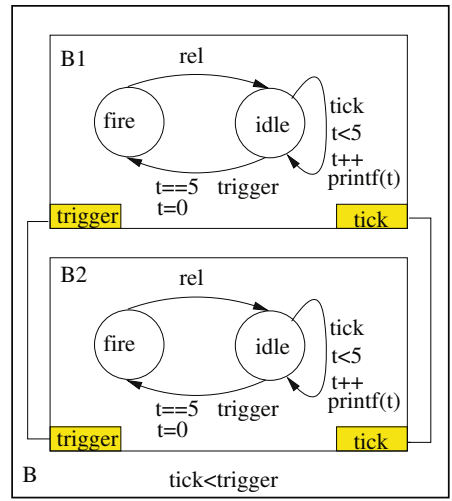

Fig. 1. A BIP model

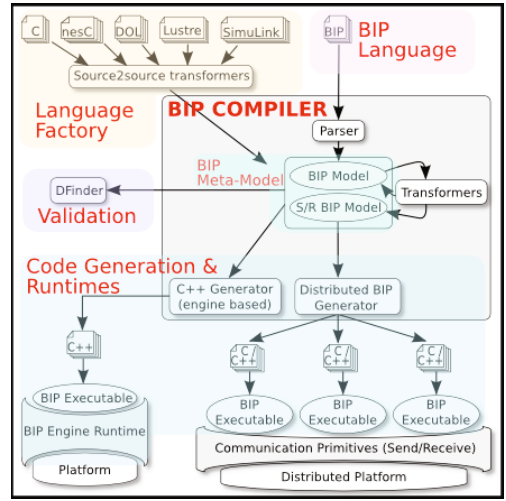

Fig. 2. BIP tools and work-flow

the interaction invariant $\Psi$, which captures constraints on the global state space induced by the synchronization. More formally, we have the following rule:

$$
\frac{\left\{B_{i}<\Phi_{i}>\right\}_{i}, \Psi \in I I\left(\|_{\gamma}\left\{B_{i}\right\}_{i},\left\{\Phi_{i}\right\}_{i}\right),\left(\bigwedge_{i} \Phi_{i}\right) \wedge \Psi \Rightarrow \Phi}{\|_{\gamma}\left\{B_{i}\right\}_{i}<\Phi>}
$$

The rule states that if all components $B_{i}$ fulfill their respective component invariants $\Phi_{i}$, the composition of all components $I I\left(\|_{\gamma}\left\{B_{i}\right\}_{i},\left\{\Phi_{i}\right\}_{i}\right)$ with the interactions $\gamma$ fulfills an interaction invariant $\Psi$, and if furthermore the conjunction of the invariants $\left(\bigwedge_{i} \Phi_{i}\right) \wedge \Psi$ implies a predicate on the global system $\Phi$, then also the global system $\|_{\gamma}\left\{B_{i}\right\}_{i}$ itself fulfills $\Phi$. In this paper we concentrate on global deadlock-freedom. Indeed, it suffices to prove the invariance of the predicate $\neg \mathcal{D I S}$, where $\mathcal{D} \mathcal{I S}$ is the set of states of the system from which all the interactions are disabled.

Tool Chain. The design flow between BIP and D-Finder is sketched in Figure2, The framework allows to (1) start from scratch and describe a composite system with the BIP language, or (2) to use the Language Factory to translate existing models described in languages such as C, DOL [15] or Simulink [13] into the BIP framework. These models then are used for validation, verification, model to model transformation and eventually generation of $\mathrm{C}++$ code for simulation or deployment. $D$-Finder plays a central role in this process to verify the initial models as well as ensuring correctness after transformation steps.

\section{$2 \quad$ D-Finder 2}

Recently, BIP has been updated and enriched with new features to improve the modeling process for building hierarchical models and add new interactions in an incremental manner. Furthermore, since the tool presentation in 4, new, more efficient techniques for computing $\Psi$ were introduced in 62 . To show the results of unifying those recent developments, this paper presents $D$-Finder 2 , the 


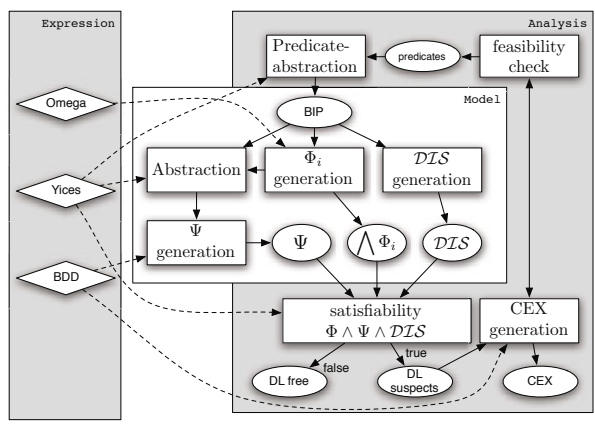

Fig. 3. Structure of the D-Finder tool

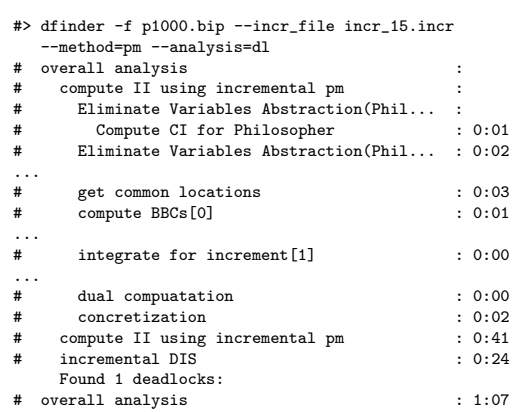

Fig. 4. Call from the command line

second edition of the D-Finder tool-set. The tool has been entirely rewritten and new techniques for computing invariants have been implemented in a modular manner.

\subsection{Computing Interaction Invariants in an Incremental Manner: the Theory behind D-Finder 2}

$D$-Finder 2 implements new efficient techniques for computing $\Psi$ that were recently introduced in 6 2. Those techniques build on the new concept of Boolean Behavioral Constraints (BBCs) that allow to relate the communication between different components with their internal transitions and hence model a unified invariant of the model. Solutions of BBCs can be used to symbolically compute a strong interaction invariant. There are two different techniques that exploit BBCs: (1) a symbolic computation based on a Fixed-Point iteration (FP), and (2) a symbolic algorithm to solve the BBCs using so called Positive Mapping (PM). Both methods allow an efficient implementation for computing interaction invariants using BDDs and show their strengths for different topologies of the model to check. The main advantage of the two aforementioned techniques is that they allow to exploit the component based design of BIP and compute interaction invariants incrementally. In the Incremental Fixed Point (IFP) and Incremental Positive Mapping (IPM) methods, D-Finder 2 partitions the model into subsystems (also called increments). The internal interactions in these subsystems are used to compute "partial" interaction invariants. Relations between different increments are considered in a second step and used to integrate the intermediate results to the final $\Psi$. Computing the global interaction invariant from smaller intermediate results allows to reduce the size of the data structures involved in the computation.

\subsection{Implementation Details}

D-Finder 2 was developed with modularity and extensibility in mind. The tool is written in Java and uses external tools and native code via the Java Native Interface (JNI) for computations. Fig. 3 gives an overview of the main modules 
of the tool. The Model block handles the parsing of the BIP code into an internal model and provides the means to compute $\Phi, \Psi$, and $\mathcal{D I S}$. Available implementations comprise the methods from the previous tool and additionally the new algorithms using fixed-point and positive mapping computation and their incremental versions. The results from Model are used from various implementations of the Analysis block, which perform further steps like the generation of possible deadlocks and, most recently, generation of counterexamples for Boolean systems (CEX). The Expression block is used by both Model and Analysis. Its main purpose is to provide an uniform interface for different back-ends that store the actual expressions. The abstraction from the back-ends allows a high degree of flexibility for implementing the algorithms. The most general implementation is a wrapper to the actual parse tree representation of the expressions (using the Eclipse Modeling Framework, EMF) and uses external tools for computations. For algorithms on Boolean variables, like computation of $\Psi$, a more succinct implementation with BDDs as back-end is used, while large systems that incorporate non-Boolean data require to directly create and maintain input files for an SMT solver on disk. These different versions of expressions can be used interchangeably in many contexts, with the respective tools being called transparently for actual computations. The Expression block also provides methods to translate between representation and manage the scopes of variables. Fig. 3 shows the use of external tools for models with non-Boolean data; for models with only Boolean variables, BDDs are used in all computation steps.

The three main blocks are complemented by a common configuration module that reads settings from default values, configuration files and command line and provides the means to instantiate the proper modules for an example to check. The used tools for SMT solving (Yices, [16]) and variable quantification (omega library, [12 are accessed using wrappers, giving rise for easy extension and replacement. Similarly, the used BDD-Manager (JavaBDD, [1]) provides a Java implementation, but has the option to use native BDD managers on supported machines. Currently we use CUDD [14 on Linux and OS X. This flexibility provides the means to develop and maintain new and experimental algorithms in the tool while leaving the main behavior intact, which is currently done, e.g., for experimental modules to perform predicate abstraction to create Boolean systems, check the reachability of deadlocks to remove false positives, and to construct error traces to understand the causes of reachable deadlocks, all of which were not present in the previous tool.

\subsection{Availability of the Tool and Example of Use}

The D-Finder 2 and BIP tools, along with the examples discussed in this paper, can be freely downloaded from [9] and 7] respectively. An excerpt of a call to D-Finder 2 for the case of dining philosophers is given in Figure 4. The example shows verification of a problem size of 1000 Philosophers partitioned into 20 increments. The first step is the computation of an abstraction to remove the variables for $\Psi$ computation (using the post conditions from $\Phi$ computation to split the states), followed by the local computations (BBC) for each of the 
Table 1. Comparison of D-Finder versions. Times in min, timeout one hour.

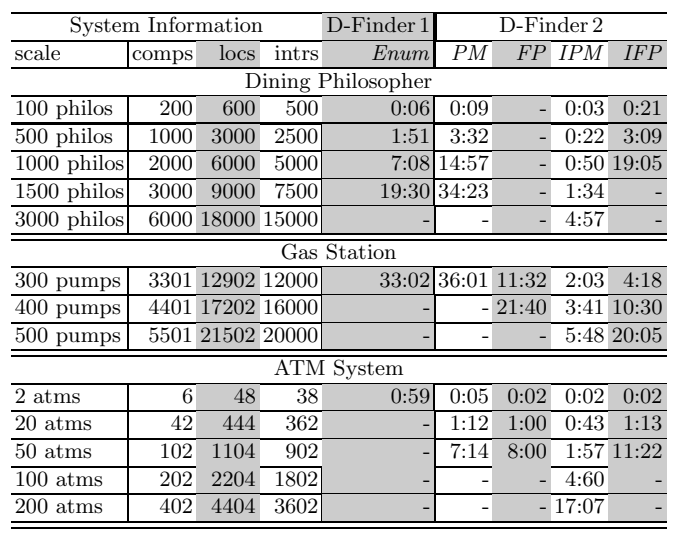

Table 2. Verification times for the Dala robot

\begin{tabular}{|c|c|c|c|c|c|c|}
\hline \multirow{2}{*}{ module } & \multirow{2}{*}{\multicolumn{3}{|c|}{ comps locs intrs }} & \multirow{2}{*}{ vars } & \multicolumn{2}{|c|}{ time } \\
\hline & & & & & D-Finder 1 & D-Finder 2 \\
\hline RFLEX & 56 & 308 & 227 & 35 & $9: 39$ & $3: 07$ \\
\hline NDD & 27 & 152 & 117 & 27 & $8: 16$ & $1: 15$ \\
\hline SICK & 43 & 213 & 202 & 29 & $1: 22$ & 1:04 \\
\hline Aspect & 29 & 160 & 117 & 21 & $0: 39$ & $0: 21$ \\
\hline Antenna & 20 & 97 & 73 & 23 & $0: 14$ & $0: 13$ \\
\hline Combined & 198 & 926 & 724 & 132 & - & $5: 05$ \\
\hline
\end{tabular}

increments and their integration. Computation of the dual and mapping to the concrete values finishes the computation of $\Psi$, which is used to directly compute the intersection with $\mathcal{D} \mathcal{I} \mathcal{S}$. Finally, the tool successfully reports one deadlock.

Large examples from real world applications may require manual assumptions on the components to rule out false positives. D-Finder 2 supports these additional inputs on the component and global level. To support organization of the required models, specifications, and output files, the tool supports so called example configuration files, which allow to collect the required files in own directories. The examples and case studies on the web site are organized in this way. The Web page of BIP [7] gives more information to introduce the language as well as details on usage and case studies. The web page of D-Finder 2 [9] comes up with illustrations of the use of the tool as well as many other case studies of huge size. The sites also reference a series of publications that give more details on the theory implemented in the tool.

\section{Experimental Results}

We compare the performance of the original version of D-Finder with the new version of the tool presented in this paper on some case studies (see 9] for more experiments). Experiments where conducted with a 32Bit Linux on Xeon $2.67 \mathrm{GHz}$ We started by considering verification of deadlock properties for the classical case studies of Dining Philosopher, the Gas Station [10], for which we assume that every pump has 10 customers, and the Automatic Teller Machine (ATM) [8]. The results are given in Table 1, where scale is the parameter of the example, comps the number of components, locs the number of control locations, and intrs the total number of interactions. The experiments where performed on Mac-Book Pro laptops with CUDD as back end for BDD computations. We see that especially the incremental versions of the new $\Psi$ computation methods led to major improvements in run time compared to the original version of the tool from [4].

To demonstrate the application of D-Finder 2 to industrial problems we want to refer to a case study on the application of BIP to autonomous robots Dala [5]. 
The case study uses software written in Genom, a tool to design modular real time software architectures, which were then translated to BIP. The translated modules implement features of the robot like movement (RFLEX), navigation (NDD) and self localization using a laser range finder (SICK), and themselves consist of more internal components, for more details see [5]. The case study shows how to use $B I P$ code generation tools and the BIP engine to create $\mathrm{C}$ code from the model, which runs at the functional level of the robot to guarantee coordination of the various modules in a correct manner. The previous tool was not able to verify this model. And while prototypes were able to show the deadlock-freedom of single modules in the past, only D-Finder 2 allowed us recently to verify the combination of all five main components. (Results are reported in Table 2). This use of the work flow of D-Finder 2 and BIP is a major change with respect to other methodologies to design autonomous robots. Indeed, most of other existing works propose functional levels that are designed manually, without any formal guarantee of correctness.

\section{References}

1. Basu, A., Bozga, M., Sifakis, J.: Modeling heterogeneous real-time components in BIP. In: SEFM, Washington, DC, USA, pp. 3-12. IEEE, Los Alamitos (2006)

2. Bensalem, S., Bogza, M., Legay, A., Nguyen, T.-H., Sifakis, J., Yan, R.: Incremental component-based construction and verification using invariants. In: FMCAD (2010)

3. Bensalem, S., Bozga, M., Nguyen, T.-H., Sifakis, J.: Compositional verification for component-based systems and application. In: Cha, S(S.), Choi, J.-Y., Kim, M., Lee, I., Viswanathan, M. (eds.) ATVA 2008. LNCS, vol. 5311, pp. 64-79. Springer, Heidelberg (2008)

4. Bensalem, S., Bozga, M., Nguyen, T.-H., Sifakis, J.: D-finder: A tool for compositional deadlock detection and verification. In: Bouajjani, A., Maler, O. (eds.) CAV 2009. LNCS, vol. 5643, pp. 614-619. Springer, Heidelberg (2009)

5. Bensalem, S., de Silva, L., Gallien, M., Ingrand, F., Yan, R.: Rock solid software: A verifiable and correct by construction controller for rover and spacecraft functional layers. In: ISAIRAS, pp. 859-866 (2010)

6. Bensalem, S., Legay, A., Nguyen, T.-H., Sifakis, J., Yan, R.: Incremental invariant generation for compositional design. In: TASE, pp. 157-167 (2010)

7. BIP tool page, http://www-verimag.imag.fr/BIP-Tools, 93.html

8. Chaudron, M.R.V., Eskenazi, E.M., Fioukov, A.V., Hammer, D.K.: A framework for formal component-based software architecting. In: SVCS (2001)

9. DFinder tool page, http://www-verimag.imag.fr/dfinder/

10. Heimbold, D., Luckham, D.: Debugging Ada tasking programs. IEEE Softw. 2(2), 47-57 (1985)

11. JavaBDD tool page, http://javabdd.sourceforge.net/

12. Omega library tool page, http://www.cs.umd.edu/projects/omega/

13. Simulink, http://www.mathworks.com/products/simulink/

14. Somenzi, F.: CUDD tool page, http://vlsi.colorado.edu/ fabio/CUDD/

15. Thiele, L., Bacivarov, I., Haid, W., Huang, K.: Mapping applications to tiled multiprocessor embedded systems. In: ACSD, pp. 29-40. IEEE, Los Alamitos (2007)

16. Yices tool page, http://yices.csl.sri.com/ 